\title{
THEORETICAL MECHANICS OF 6-DIMENSIONAL (6D) CENTRAL-FORCE MOTION WITH TANGENTIAL OSCILLATIONS.
}

\author{
${ }^{1}$ Edison A. Enaibe, ${ }^{2}$ Akpata Erhieyovwe and ${ }^{3}$ Osafile E. Omosede \\ 1,3 Department of Physics, Federal University of Petroleum Resources \\ P. M. B. 1221, Effurun, Nigeria. Phone number : $+2348068060786,+23408074229081$, \\ E-mail: aroghene70@yahoo.com \\ ${ }^{2}$ Department of Physics, University of Benin, P. M. B. 1154, Benin City, Edo State, Nigeria. \\ Phone number: +2348024515959, E-mail:akpataleg@hotmail.com
}

NOTE: All correspondence should be directed to the second author;

\begin{abstract}
The relevance of the Central - force motion in the macroscopic and microscopic frames warrants a detailed study of the theoretical mechanics associated with it. So far, researchers have only considered central - force motion, as motion only in the translational and rotational elliptical plane with polar coordinates $(r, \theta)$. However, the theoretical knowledge advanced by these researchers in line with this type of motion is scientifically restricted as several possibilities are equally applicable. In order to make the mechanics of a Central - force motion sufficiently meaningful, we have in this work extended the theory which has only been that of translational and rotational in the elliptical plane, by including fictitious radii and spin oscillations $\left(r_{1}, r_{2} ; \beta, \alpha, \phi, \mu\right)$ of the body about the axis of rotation. In this work, we used the methods of Newtonian mechanics to establish the new central-force field obeyed by the motion of a body, when the effect of spin oscillation is added. The new central-force field comprises of the radial accelerations, translational orbital angular velocity and the oscillating spin angular velocities. The energy conveyed in the spin oscillating phase increases as the orbital oscillating angles above or below the horizon of the elliptical plane is increased.
\end{abstract}

Keywords: Elliptical plane, fictitious radii, orbital radial oscillating angles, tangential spin oscillating angles.

Academic Discipline: Health Science.

Subject Classification: TheoreticalPhysics

\section{Council for Innovative Research}

Peer Review Research Publishing System

Journal: JOURNAL OF ADVANCES IN NATURAL SCIENCES

Vol 1, No. 1

editorjansonline@gmail.com

www.cirworld.com 


\subsection{Introduction}

The forces of nature are unbalanced and therefore are in a state of non-equilibrium. For instance, the gravitational force is not uniform but it varies from one region of space to another. The difference in elliptic motion of the planets around the perihelion and aphelion is a consequence of the non-uniform and unbalanced nature of this force.

A body undergoing a central force motion in an unbounded region of space induces electrostatic potential on the path of its motion. This potential creates a dipole moment which tends to oscillate the body up and down about a given equilibrium position. Consequent upon this, the body would certainly have a definite memory of the repeated path and directions of motion even in the absence of any immediate directional driving force. The up and down oscillation of a body in a central force field, with example the earth could be what is responsible for the several phenomena such as the solitary wave, tidal wave and ocean current experience in nature.

The fundamental forces of nature depend only on the distance from the source. All complex interactions that occur in the real world arise from these forces, and while many of them are usually described in a more complex manner, their origin can be found in the fundamental forces that depend only on distance. It is the presence of many sources of the distancedependent forces that enables the complex world we know to exist [1]. For motion that is confined to a plane defined by the action of a central force, the logical choice of a coordinate frame is polar coordinates with the center of the force field located at the origin of the coordinate system.

In some cases, the nature of the force attracting the motion of a body to a central -point, could make the body oscillate up and down about its mean equilibrium position. The added effects of the up and down spin oscillations will cause the body to have a different generalized coordinates apart from the one of translational and rotational elliptical plane motion. The only requirement for the generalized coordinates is that they span the space of the motion and be linearly independent.

Central - force motion is the motion which takes place under the action of a force that is always directed towards or away from a focus [2]. Perhaps, a good example of this type of motion is that of the planets round the sun. Nuclear forces binding electrons to an atom undoubtedly have a central character. The relevance of the Central - force motion in the macroscopic and microscopic frames warrants a detailed study of the theoretical mechanics associated with it.

So far, researchers have only considered central - force motion, as motion only in the translational and rotational plane with polar coordinates $(r, \theta)$, for example, see Keplerian orbits [3, 4]. However, the theoretical knowledge advanced by these researchers in line with this type of motion is scientifically restricted as several possibilities are equally applicable.

Some of the conditions satisfied by a body undergoing a Central - force motion is as follows: (i) the motion of the body can be translational and rotational in the elliptical plane represented by the polar coordinates $(r, \theta)$, (ii) the body can be rotating and revolving about its own axis in the elliptical plane $(r, \theta)$, (iii) the body can be translating and rotating in the elliptical plane $(r, \theta)$, at the same time, oscillating up and down about its own axis (iv) the body can be translating and rotating in the elliptical plane $(r, \theta)$, at the same time, oscillating up and down but not below its axis of rotation (v) the combination of any of these conditions form another class of a Central - force motion [5].

In order to make the mechanics of a Central - force motion sufficiently meaningful, we have in this work extended the theory which has only been that of translational and rotational in the elliptical plane with polar coordinates $(r, \theta)$, by including vertical spin oscillations. This circumstance, we shall be contending with a total of 6 - generalized coordinates or degrees of freedom; 2 from the translational and rotational motion in the elliptical plane $(r, \theta), 2$ from the orbital spin oscillations $(\beta, \alpha)$ and 2 from the tangential spin oscillations $(\mu, \phi)$. Consequently, these parameters form the basis of our classical theory of 6 -dimensional motion [6].

The number of independent ways in which a mechanical system can move without violating any constraints which may be imposed is called the number of degrees of freedom of the system. The number of degrees of freedom is the number of quantities which must be specified in order to determine the velocities of all particles in the system for any motion which does not violate the constraints [7].

There is a single source producing the force that depends only on distance in the theory of central-force motion and the force law is symmetric [8]. If this is the case, then, there can be no torques present in the system as there would have to be a preferred axis about which the torques acts.

In this work, we are solving the problem of oscillating central force motion in a resistive non-symmetric system. That is, the upward displacement is not equal to the downward displacement in the tangential spin oscillating phase. Consequently, the radii distances from the central point are not equal. This however, causes torques thereby making the system under study non-spherically symmetric.

This paper is outlined as follows. Section 1, illustrates the basic concept of the work under study. The mathematical theory is presented in section 2 . While in section 3 , we present the analytical discussion of the results obtained. The conclusion of this work is shown in section 4 and this is immediately followed by appendix and list of references. 


\section{$1.1 \quad$ Research methodology}

The analytical geometry of the work is first figuratively represented. This provides the pictorial understanding of the work under investigation and the possibility of specifying the required generalized coordinates. Thereafter, simple rule of trigonometry was used to define the vector quantities which we need for the evaluation of the physical quantities. Finally, differential techniques in combination with the theory of classical mechanics are utilized in the discussion of the problem of $6 \mathrm{D}$ motion.

\subsection{Mathematical Theory}

\subsection{Evaluation of the 6-D central - force field}

We have elaborately shown in (A.6) that the position vector $\vec{r}$ of a body whose motion is translational and rotational in the elliptic orbit, as well as oscillating about a given equilibrium position in a central-force field is given by the equation

$$
\begin{aligned}
& \vec{r}=r \hat{r}(\theta)+r_{1} \hat{r}_{1}(\beta, \mu)+r_{2} \hat{r}_{2}(\alpha, \phi) \\
& v=\frac{d \vec{r}}{d t}=\frac{d r}{d t} \hat{r}+r \frac{d \hat{r}}{d \theta} \frac{d \theta}{d t}+\frac{d r_{1}}{d t} \hat{r}_{1}+r_{1}\left(\frac{d \hat{r}_{1}}{d \mu} \frac{d \mu}{d t}+\frac{d \hat{r}_{1}}{d \beta} \frac{d \beta}{d t}\right)+\frac{d r_{2}}{d t} \hat{r}_{2}+r_{2}\left(\frac{d \hat{r}_{2}}{d \phi} \frac{d \phi}{d t}+\frac{d \hat{r}_{2}}{d \alpha} \frac{d \alpha}{d t}\right) \\
& v=\dot{r} \hat{r}+r \dot{\theta} \hat{\theta}+\dot{r}_{1} \hat{r}_{1}+r_{1} \dot{\mu} \hat{\mu}+r_{1} \dot{\beta} \hat{\beta}+\dot{r}_{2} \hat{r}_{2}+r_{2} \dot{\phi} \hat{\phi}+r_{2} \dot{\alpha} \hat{\alpha} \\
& a=\frac{d^{2} \vec{r}}{d t^{2}}=\frac{d v}{d t}=\ddot{r} \hat{r}+\dot{r} \dot{\theta}+\dot{r} \dot{\theta}+r \ddot{\theta} \hat{\theta}+r \dot{\theta}^{2} \frac{d \hat{\theta}}{d \theta}+\ddot{r}_{1} \hat{r}_{1}+\dot{r}_{1}(\dot{\mu} \hat{\mu}+\dot{\beta} \hat{\beta})+ \\
& \dot{r}_{1} \dot{\mu} \hat{\mu}+r_{1} \ddot{\mu} \hat{\mu}+r_{1} \dot{\mu}^{2} \frac{d \hat{\mu}}{d \mu}+\dot{r}_{1} \dot{\beta} \hat{\beta}+r_{1} \ddot{\beta} \hat{\beta}+r_{1} \dot{\beta}^{2} \frac{d \hat{\beta}}{d \beta}+\ddot{r}_{2} \hat{r}_{2}+\dot{r}_{2}(\dot{\phi} \hat{\phi}+\dot{\alpha} \hat{\alpha})+\dot{r}_{2} \dot{\phi} \hat{\phi}+ \\
& r_{2} \ddot{\phi} \hat{\phi}+r_{2} \dot{\phi}^{2} \frac{d \hat{\phi}}{d \phi}+\dot{r}_{2} \dot{\alpha} \hat{\alpha}+r_{2} \ddot{\alpha} \hat{\alpha}+r_{2} \dot{\alpha}^{2} \frac{d \hat{\alpha}}{d \alpha} \\
& a=\left(\ddot{r}-r \dot{\theta}^{2}\right) \hat{r}+(r \ddot{\theta}+2 \dot{r} \dot{\theta}) \hat{\theta}+\left(\ddot{r}_{1}-r_{1} \dot{\mu}^{2}\right) \hat{r}_{1}+\left(r_{1} \ddot{\mu}+2 \dot{r}_{1} \dot{\mu}\right) \hat{\mu}+\left(r_{1} \ddot{\beta}+2 \dot{r}_{1} \dot{\beta}+2 r_{1} \dot{\beta}^{2} \tan \beta\right) \hat{\beta}+ \\
& \left(\ddot{r_{2}}-r_{2} \dot{\phi}^{2}\right) \hat{r}_{2}+\left(r_{2} \ddot{\phi}+2 \dot{r}_{2} \dot{\phi}\right) \hat{\phi}+\left(r_{2} \ddot{\alpha}+2 \dot{r}_{2} \dot{\alpha}+2 r_{2} \dot{\alpha}^{2} \tan \alpha\right) \hat{\alpha}
\end{aligned}
$$

Equation (2.3) and (2.5) are the new velocity and acceleration equations which govern the motion of a body undergoing a central-force motion when the effect of vertical oscillation is added. They are both six-dimensional (6D) in character. But while the velocity is only translational with respect to the generalized coordinates, the acceleration also contains an oscillating phase.

In a central force motion, the angular momentum is constant, because the torque exerted by the force is zero. As a consequence, the body moves on a plane perpendicular to the angular momentum vector containing the origin, and obeys Kepler's second law. If the angular momentum is zero, the body moves along the line joining it with the origin.

As a result, let us disengage the acceleration equation in (2.5) with the view that the $5^{\text {th }}$ and the $8^{\text {th }}$ terms contain the elements of angular momentum and orbital oscillating phases. Thus

$$
\begin{aligned}
& a=\left(\ddot{r}-r \dot{\theta}^{2}\right) \hat{r}+(r \ddot{\theta}+2 \dot{r} \dot{\theta}) \hat{\theta}+\left(\ddot{r}_{1}-r_{1} \dot{\mu}^{2}\right) \hat{r}_{1}+\left(r_{1} \ddot{\mu}+2 \dot{r}_{1} \dot{\mu}\right) \hat{\mu}+\left(r_{1} \ddot{\beta}+2 \dot{r} \dot{\beta}\right) \hat{\beta}+\left(2 r_{1} \dot{\beta}^{2} \tan \beta\right) \hat{\beta}+ \\
& \left(\ddot{r}_{2}-r_{2} \dot{\phi}^{2}\right) \hat{r}_{2}+\left(r_{2} \ddot{\phi}+2 \dot{r}_{2} \dot{\phi}\right) \hat{\phi}+\left(r_{2} \ddot{\alpha}+2 \dot{r}_{2} \dot{\alpha}\right) \hat{\alpha}+\left(2 r_{2} \dot{\alpha}^{2} \tan \alpha\right) \hat{\alpha}
\end{aligned}
$$

In classical mechanics, a central force is a force whose magnitude only depends on the distance $r$, of the body from the origin and is directed along the line joining them [7]. Thus, from the analytical geometry of the central-force motion shown in fig. A. 1 , in the appendix, permits us to write in terms of vector algebra that

$$
F(r)=f(\|r\|)\left(\hat{r}, \hat{r}_{1}, \hat{r}_{2} ; \hat{\beta}, \hat{\alpha}\right)=f(\|r\|) \hat{r}+f\left(\left\|r_{1}\right\|\right) \hat{r}_{1}+f\left(\left\|r_{2}\right\|\right) \hat{r}_{2}+f\left(\left\|r_{1}\right\|\right) \hat{\beta}+f\left(\left\|r_{2}\right\|\right) \hat{\alpha}=m a
$$

where $F$ is a vector valued force function, $f$ is a scalar valued force function, $r$ is the position vector, $\|r\|$ is its length, and $\hat{r}=r /\|r\|$, is the corresponding unit vector. 
We can simply convert (2.6) to force by multiplying through it by the mass $m$ of the body and equate the resulting expression to (2.7). Note that we are utilizing the orbital oscillating phase in (2.6) which is acting in the directions of $\hat{\beta}$ and $\hat{\alpha}$ in the force calculation. We shall separately utilize the non-oscillating phase which is acting in the directions of $\hat{\beta}$ and $\hat{\alpha}$ in the calculation of the angular momentum. As a consequence, we obtain the following sets of canonical equations of the motion.

$$
\begin{aligned}
& f(r)=m\left\{\left(\ddot{r}-r \dot{\theta}^{2}\right)+\left(\ddot{r}_{1}-r_{1} \dot{\mu}^{2}\right)+\left(\ddot{r}_{2}-r_{2} \dot{\phi}^{2}\right)+2\left(r_{1} \dot{\beta}^{2} \tan \beta+r_{2} \dot{\alpha}^{2} \tan \alpha\right)\right\} \\
& m(r \ddot{\theta}+2 \dot{r} \dot{\theta})=0 \\
& m\left(r_{1} \ddot{\mu}+2 \dot{r}_{1} \dot{\mu}\right)=0 \\
& m\left(r_{1} \ddot{\beta}+2 \dot{r_{1}} \dot{\beta}\right)=0 \\
& m\left(r_{2} \ddot{\alpha}+2 \dot{r}_{2} \dot{\alpha}\right)=0 \\
& m\left(r_{2} \ddot{\phi}+2 \dot{r}_{2} \dot{\phi}\right)=0
\end{aligned}
$$

The sets of equation (2.9) - (2.13) determines the angular momentum which are the constants of the motion in the direction of increasing coordinates, $\theta, \beta, \mu, \alpha$ and $\phi$.

Equation (2.8) is the required $6 \mathrm{D}$ central-force field which we have developed in this study. It governs the motion of a body undergoing a central-force motion when the effect of spin oscillation is added.

\subsection{Evaluation of the Oscillating Energy $E_{o s c}$ and the Orbital Spin Oscillating Velocities.}

Let us discuss some possibilities associated with the new acceleration equation given by (2.5). The orbital spin oscillating acceleration takes place in the directions of increasing $\hat{\beta}$ and $\hat{\alpha}$. At position $D$ (see fig. A. 1 in the appendix), when $\alpha=0$, then the orbital spin oscillating acceleration acting in the direction of increasing $\hat{\beta}$ becomes applicable. It describes the motion in the upper orbital oscillating frame, above $\mathrm{C}$. At position $\mathrm{B}, \beta=0$, then the orbital spin oscillating acceleration acting in the direction of increasing $\hat{\alpha}$ becomes applicable. It describes the motion in the lower orbital oscillating frame, below $\mathrm{C}$. As a consequence

$$
\begin{array}{ll}
\left(r_{1}^{2} \ddot{\beta}+2 r_{1} \dot{r}_{1} \dot{\beta}+2 r_{1}^{2} \dot{\beta}^{2} \tan \beta\right)=0 & ;\left(m / r_{1} \neq 0, \alpha=0\right) \\
\left(r_{2}^{2} \ddot{\alpha}+2 r_{2} \dot{r}_{2} \dot{\alpha}+2 r_{2}^{2} \dot{\alpha}^{2} \tan \alpha\right)=0 & ;\left(m / r_{2} \neq 0, \beta=0\right)
\end{array}
$$

The simultaneous nature of the two orbital spin oscillating equations for both the upper and the lower frames would enable us to use one of them as case study and assume the same result for the other. Hence, equation (2.14) can be compactly written as

$$
\begin{aligned}
& \frac{d}{d t}\left(r_{1}^{2} \dot{\beta}\right)+\frac{d}{d t} \int 2 r_{1}^{2} \dot{\beta}^{2} \tan \beta=0 \\
& \frac{d}{d t}\left(r_{1}^{2} \dot{\beta}+\int 2 r_{1}^{2} \dot{\beta}^{2} \tan \beta\right)=0 \\
& r_{1}^{2} \dot{\beta}+\int 2 r_{1}^{2} \dot{\beta}^{2} \tan \beta=E_{1} \quad ; \quad\left(0 \prec \beta \prec 90^{0}\right)
\end{aligned}
$$

with a similar expression for (2.15) as

$$
r_{2}^{2} \dot{\alpha}+\int 2 r_{2}^{2} \dot{\alpha}^{2} \tan \alpha=E_{2} \quad ; \quad\left(0 \prec \alpha \prec 90^{\circ}\right)
$$

where $E_{1}$ and $E_{2}$ are the constants of the motion associated with the fictitious radii $r_{1}$ and $r_{2}$, whose values are respectively the functions of the orbital oscillating angles $\beta$ and $\alpha$. 
$E(\beta, \alpha)=r_{1}^{2} \dot{\beta}+\int 2 r_{1}^{2} \dot{\beta}^{2} \tan \beta+r_{2}^{2} \dot{\alpha}+\int 2 r_{2}^{2} \dot{\alpha}^{2} \tan \alpha$

Hence, the oscillating energy posses by the body in terms of $\beta$ and $\alpha$ in the oscillating phase is given by (2.20). This equation determines how energy is conveyed up and down in the oscillating phase.

It can be interpreted that equation (2.14) is quadratic in $\dot{\beta}$ and accordingly, we can write that

$\dot{\beta}^{2}+\left(\frac{\dot{r}_{1}}{r_{1} \tan \beta}\right) \dot{\beta}+\frac{\ddot{\beta}}{2 \tan \beta}=0$

$\dot{\beta}=\frac{-2 \dot{r}_{1}}{r_{1} \tan \beta} \pm \frac{2 \dot{r}_{1}}{r_{1} \tan \beta}\left(\sqrt{1-\frac{2 r_{1}^{2}}{\dot{r}_{1}^{2}} \ddot{\beta} \tan \beta}\right)$

Following the same algebraic subroutine we obtain a similar expression for $\dot{\alpha}$ using (2.15) as

$\dot{\alpha}=\frac{-2 \dot{r}_{2}}{r_{2} \tan \alpha} \pm \frac{2 \dot{r}_{2}}{r_{2} \tan \alpha}\left(\sqrt{1-\frac{2 r_{2}^{2}}{\dot{r}_{2}^{2}} \ddot{\alpha} \tan \alpha}\right)$

The discriminate of the equations for $\dot{\beta}$ and $\dot{\alpha}$ are both respectively zero provided

$\dot{r}_{1}=\sqrt{2 r_{1}^{2} \ddot{\beta} \tan \beta}$

$\dot{r}_{2}=\sqrt{2 r_{2}^{2} \ddot{\alpha} \tan \alpha}$

Equations (2.24) and (2.25), show that the fictitious radii velocity $\dot{r}_{1}$ and $\dot{r}_{2}$ increases as the orbital oscillating angles $\beta$ and $\alpha$ is increased.

\subsection{Evaluation of the Lagrange's Equations of Motion.}

From equation (2.3) we realize that the kinetic energy $T$ of the body can be written as

$$
\begin{aligned}
& T=\frac{1}{2} m\left(\dot{r}^{2}+r^{2} \dot{\theta}^{2}+\dot{r}_{1}^{2}+r_{1}^{2} \dot{\mu}^{2}+r_{1}^{2} \dot{\beta}^{2}+\dot{r}_{2}^{2}+r_{2}^{2} \dot{\phi}^{2}+r_{2}^{2} \dot{\alpha}^{2}\right) \\
& L=T-V(r) \\
& \frac{d}{d t}\left(\frac{\partial L}{\partial \dot{q}_{k}}\right)-\frac{\partial L}{\partial q_{k}}=0 \\
& \frac{d}{d t}\left(\frac{\partial}{\partial \dot{q}_{k}}(T-V(r))\right)-\frac{\partial}{\partial q_{k}}(T-V(r))=0 \\
& \frac{d}{d t}\left(\frac{\partial T}{\partial \dot{q}_{k}}-\frac{\partial V(r)}{\partial \dot{q}}\right)-\frac{\partial T}{\partial q_{k}}+\frac{\partial V(r)}{\partial q_{k}}=0 \\
& \frac{\partial}{\partial \dot{q}_{k}}(V(r))=0 ; \quad \frac{\partial V(r)}{\partial q_{k}}=\frac{d V}{d q_{k}} \\
& \frac{d}{d t}\left(\frac{\partial T}{\partial \dot{q}_{k}}\right)-\frac{\partial T}{\partial q_{k}}+\frac{d V(r)}{d q_{k}}=0 \\
& q_{k}=\left(r, \theta, r_{1}, \beta, \mu, r_{2}, \alpha, \phi\right) ; \quad \dot{q}_{k}=\left(\dot{r}, \dot{\theta}, \dot{r}_{1}, \dot{\beta}, \dot{\mu}, \dot{r}_{2}, \dot{\alpha}, \dot{\phi}\right)
\end{aligned}
$$


where $q_{k}$ are the generalized coordinates, $\dot{q}_{k}$ are the associated velocity counterparts, $\partial L / \partial q_{k}$ generalized velocity, $\partial L / \partial \dot{q}_{k}$ generalized momentum.

The Lagrange's equations of motion are thus generally given as follows.

$\frac{d}{d t}(m \dot{r})-\left(m r \dot{\theta}^{2}\right)+\frac{d V}{d r}=0$

$\frac{d}{d t}\left(m \dot{r}_{1}\right)-\left(m r_{1} \dot{\mu}^{2}\right)-\left(m r_{1} \dot{\beta}^{2}\right)+\frac{d V_{1}}{d r_{1}}=0$

$\frac{d}{d t}\left(m \dot{r}_{2}\right)-\left(m r_{2} \dot{\phi}^{2}\right)-\left(m r_{2} \dot{\alpha}^{2}\right)+\frac{d V_{2}}{d r_{2}}=0$

We know from (2.9) that

$$
\begin{aligned}
& m(r \ddot{\theta}+2 \dot{r} \dot{\theta})=0 ; \quad \frac{m}{r}\left(r^{2} \ddot{\theta}+2 r \dot{r} \dot{\theta}\right)=0 \quad ; \quad m\left(r^{2} \ddot{\theta}+2 r \dot{r} \dot{\theta}\right)=0 \quad ;(1 / r \neq 0) \\
& \frac{d}{d t}\left(m r^{2} \dot{\theta}\right)=0 ; \quad\left(m r^{2} \dot{\theta}\right)=K \quad ; \quad \dot{\theta}=\frac{K}{m r^{2}} \quad ; \dot{\theta}^{2}=\frac{K^{2}}{m^{2} r^{4}}
\end{aligned}
$$

Following the same algebraic subroutine we obtain the following angular momentum equations associated with the other generalized coordinates. Since

$$
\begin{aligned}
& \frac{\partial T}{\partial q_{k}}=0 \quad ; \quad \frac{d V(r)}{d q_{k}}=0 \\
& \frac{d}{d t}\left(m r_{1}^{2} \dot{\mu}\right)=0 ; \quad\left(m r_{1}^{2} \dot{\mu}\right)=K_{1} \quad ; \quad \dot{\mu}=\frac{K_{1}}{m r_{1}^{2}} \quad ; \dot{\mu}^{2}=\frac{K_{1}^{2}}{m^{2} r_{1}^{4}} \\
& \frac{d}{d t}\left(m r_{1}^{2} \dot{\beta}\right)=0 ; \quad\left(m r_{1}^{2} \dot{\beta}\right)=K_{1} \quad ; \quad \dot{\beta}=\frac{K_{1}}{m r_{1}^{2}} \quad ; \dot{\beta}^{2}=\frac{K_{1}^{2}}{m^{2} r_{1}^{4}} \\
& \frac{d}{d t}\left(m r_{2}^{2} \dot{\alpha}\right)=0 ; \quad\left(m r_{2}^{2} \dot{\alpha}\right)=K_{2} \quad ; \quad \dot{\alpha}=\frac{K_{2}}{m r_{2}^{2}} \quad ; \dot{\alpha}^{2}=\frac{K_{2}^{2}}{m^{2} r_{2}^{4}} \\
& \frac{d}{d t}\left(m r_{2}^{2} \dot{\phi}\right)=0 ; \quad\left(m r_{2}^{2} \dot{\phi}\right)=K_{2} \quad ; \quad \dot{\phi}=\frac{K_{2}}{m r_{2}^{2}} \quad ; \dot{\phi}^{2}=\frac{K_{2}^{2}}{m^{2} r_{2}^{4}}
\end{aligned}
$$

By replacing equations (2.38) - (2.43) into (2.34), (2.35) and (2.36) respectively and noting that the Lagrange's equations in the case of a conservative field is

$$
\begin{aligned}
& \nabla x F=0 \quad ; \quad \frac{d V}{d r}=-f(r) \\
& \frac{d}{d t}(m \dot{r})-\left(\frac{K^{2}}{m r^{3}}\right)-f(r)=0 \\
& \frac{d}{d t}\left(m \dot{r}_{1}\right)-\left(\frac{K_{1}^{2}}{m r_{1}^{3}}\right)-\left(\frac{K_{1}^{2}}{m r_{1}^{3}}\right)-f\left(r_{1}\right)=0
\end{aligned}
$$




$$
\frac{d}{d t}\left(m \dot{r}_{2}\right)-\left(\frac{K_{2}^{2}}{m r_{2}^{3}}\right)-\left(\frac{K_{2}^{2}}{m r_{2}^{3}}\right)-f\left(r_{2}\right)=0
$$

Multiply through (2.45), (2.46) and (2.47) by $\dot{r}, \dot{r}_{1}$ and $\dot{r}_{2}$ respectively and integrate, we get

$$
\begin{aligned}
& \int \frac{d}{d t}(m \dot{r}) \dot{r}-\int\left(\frac{K^{2}}{m r^{3}}\right) \dot{r}-\int f(r) \dot{r}=\int 0 \\
& \int \frac{d}{d t}\left(m \dot{r}_{1}\right) \dot{r}_{1}-\int\left(\frac{K_{1}^{2}}{m r_{1}^{3}}\right) \dot{r}_{1}-\int\left(\frac{K_{1}^{2}}{m r_{1}^{3}}\right) \dot{r}_{1}-\int f\left(r_{1}\right) \dot{r}_{1}=\int 0 \\
& \int \frac{d}{d t}\left(m \dot{r}_{2}\right) \dot{r}_{2}-\int\left(\frac{K_{2}^{2}}{m r_{2}^{3}}\right) \dot{r}_{2}-\int\left(\frac{K_{2}^{2}}{m r_{2}^{3}}\right) \dot{r}_{2}-\int f\left(r_{2}\right) \dot{r}_{2}=\int 0
\end{aligned}
$$

So that we obtain separately for (2.48), (2.49) and (2.50) the below consecutive equations

$$
\begin{aligned}
& \left(\frac{1}{2} m \dot{r}^{2}\right)-\left(\frac{K^{2}}{m r^{2}}\right)-f(r) r=E \\
& \left(\frac{1}{2} m \dot{r}_{1}^{2}\right)-\left(\frac{K_{1}^{2}}{m r_{1}^{2}}\right)-\left(\frac{K_{1}^{2}}{m r_{1}^{2}}\right)-f\left(r_{1}\right) r_{1}=E_{1} \\
& \left(\frac{1}{2} m \dot{r}_{2}^{2}\right)-\left(\frac{K_{2}^{2}}{m r_{2}^{2}}\right)-\left(\frac{K_{2}^{2}}{m r_{2}^{2}}\right)-f\left(r_{2}\right) r_{2}=E_{2} \\
& E_{r}=E+E_{1}+E_{2} \\
& E_{r}=\frac{1}{2} m\left(\dot{r}^{2}+\dot{r}_{1}^{2}+\dot{r}_{2}^{2}\right)-\left(\frac{K^{2}}{m r^{2}}\right)-2\left(\frac{K_{1}^{2}}{m r_{1}^{2}}\right)-2\left(\frac{K_{2}^{2}}{m r_{2}^{2}}\right)-f(r) r-f\left(r_{1}\right) r_{1}-f\left(r_{2}\right) r_{2}
\end{aligned}
$$

Thus equation (2.55) is the equation of the total radial energy $E_{r}$ possess by the body with respect to the three independent radii paths.

\subsection{Evaluation of the (6D) Area Velocity for a Central Force Field.}

The total area covered by the body is therefore the sum of the differential area between two radii in the elliptic plane and the two triangular areas in the vertical oscillating plane, with $r$ as the common base of the two triangles. Thus the time derivative of the total area covered by the body gives the areal velocity. We should note that the body will transvers twice the area of the triangle because of the to and fro oscillating motion in the upper and lower elliptic planes. Hence

$$
\begin{aligned}
& A=\frac{1}{2} r^{2} \theta+r P+p Q=\frac{1}{2} r^{2} \theta+r\left(r_{1} \sin \mu \tan \beta\right)+r\left(r_{2} \sin \phi \tan \alpha\right) \\
& \left(2.5 \frac{d A}{d t}=\frac{1}{2} r^{2} \frac{d \theta}{d t}+r r_{1}\left(\frac{d(\sin \mu)}{d \mu} \frac{d \mu}{d t} \tan \beta+\frac{d(\tan \beta)}{d \beta} \frac{d \beta}{d t} \sin \mu\right)+r r_{2}\left(\frac{d(\sin \phi)}{d \phi} \frac{d \phi}{d t} \tan \alpha+\frac{d(\tan \alpha)}{d \alpha} \frac{d \alpha}{d t} \sin \phi\right)\right. \\
& \frac{d A}{d t}=\frac{1}{2} r^{2} \dot{\theta}+r r_{1}\left(\cos \mu \tan \beta \dot{\mu}+\sec ^{2} \beta \sin \mu \dot{\beta}\right)+r r_{2}\left(\cos \phi \tan \alpha \dot{\phi}+\sec ^{2} \alpha \sin \phi \dot{\alpha}\right) \\
& \frac{d A}{d t}=\frac{1}{2} r^{2}\left(\frac{k}{m r^{2}}\right)+r r_{1}\left(\cos \mu \tan \beta\left(\frac{k_{1}}{m r_{1}^{2}}\right)+\sec ^{2} \beta \sin \mu\left(\frac{k_{1}}{m r_{1}^{2}}\right)\right)+r r_{2}\left(\cos \phi \tan \alpha\left(\frac{k_{2}}{m r_{2}^{2}}\right)+\sec ^{2} \alpha \sin \phi\left(\frac{k_{2}}{m r_{2}^{2}}\right)\right)
\end{aligned}
$$


$\left.\frac{d A}{d t}=\frac{1}{2}\left(\frac{k}{m}\right)+\frac{r}{r_{1}}\left(\frac{k_{1}}{m}\right)\left(\cos \mu \tan \beta+\sec ^{2} \beta \sin \mu\right)\right)+\frac{r}{r_{2}}\left(\frac{k_{2}}{m}\right)\left(\cos \phi \tan \alpha+\sec ^{2} \alpha \sin \phi\right)$

$\left.\frac{d A}{d t}=\frac{1}{2}\left(\frac{k}{m}\right)+\left(\frac{k_{1}}{m}\right)\left(\sin \mu \cos \mu \tan \beta+\sec ^{2} \beta \sin ^{2} \mu\right)\right)+\left(\frac{k_{2}}{m}\right)\left(\sin \phi \cos \phi \tan \alpha+\sec ^{2} \alpha \sin ^{2} \phi\right)$

Since $r / r_{1}=\sin \mu$ and $r / r_{2}=\sin \phi$, see appendix (A.1).We know from the geometry of fig. A. 1 , that the fictitious radii $r_{1}$ and $r_{2}$ are approximately equal to one another and they are both assumed to be slightly greater than the elliptical radius $r$. However, in a more realistic model the displacement of the body from the mean equilibrium position is the same for both the upper and lower regions of the ellipse then $\alpha=\beta, \phi=\mu$ and $k_{2}=k_{1}=k$, and as a result

$\left.\frac{d A}{d t}=\frac{1}{2}\left(\frac{k}{m}\right)+2\left(\frac{k_{1}}{m}\right)\left(\sin \mu \cos \mu \tan \beta+\sec ^{2} \beta \sin ^{2} \mu\right)\right)$

$\left.\frac{d A}{d t}=\frac{1}{2}\left(\frac{k}{m}\right)\left(1+4\left(\sin \mu \cos \mu \tan \beta+\sec ^{2} \beta \sin ^{2} \mu\right)\right)\right)$

\subsection{The Inverse Square Law of Central Force Field.}

Since the body moves in a central force field, then the potential energy is $U=-\frac{k}{r}-m g P-m g Q$

So that the time average of the potential energy is

$\langle U\rangle=-\frac{1}{\tau} \int_{0}^{r} \frac{k}{r} d t-m g \frac{1}{\tau_{1}} \int_{0}^{r_{1}}\left(r_{1} \sin \mu \tan \beta\right) d t_{1}-m g \frac{1}{\tau_{2}} \int_{0}^{r_{2}}\left(r_{2} \sin \phi \tan \alpha\right) d t_{2}$

From (2.24) and (2.25)

$\frac{d r_{1}}{d t_{1}}=\sqrt{2 r_{1}^{2} \ddot{\beta} \tan \beta} \Rightarrow d t_{1}=\frac{d r_{1}}{r_{1} \sqrt{2 \ddot{\beta} \tan \beta}}$

$\frac{d r_{2}}{d t_{2}}=\sqrt{2 r_{2}^{2} \ddot{\alpha} \tan \alpha} \Rightarrow d t_{2}=\frac{d r_{2}}{r_{2} \sqrt{2 \ddot{\alpha} \tan \alpha}}$

We observe from(2.38), (2.40) and (2.41) that since the motion is a central motion, the angular momentum is a constant of motion, as a result

$$
\begin{aligned}
& \left(m r^{2} \dot{\theta}\right)=\ell=\text { const. } \Rightarrow d t=\frac{m r^{2}}{\ell} d \theta \\
& \dot{\mu}=\frac{k_{1}}{m r_{1}^{2}} \Rightarrow \frac{d \mu}{d t}=\frac{k_{1}}{m r_{1}^{2}} \Rightarrow d \mu=\frac{k_{1}}{m r_{1}^{2}} d t \Rightarrow \mu=\frac{k_{1}}{m r_{1}^{2}} t_{1} \Rightarrow t_{1}=\frac{m r_{1}^{2} \mu}{k_{1}} \\
& \dot{\beta}=\frac{k_{1}}{m r_{1}^{2}} \Rightarrow \frac{d \beta}{d t}=\frac{k_{1}}{m r_{1}^{2}} \Rightarrow d \beta=\frac{k_{1}}{m r_{1}^{2}} d t \Rightarrow \beta=\frac{k_{1}}{m r_{1}^{2}} t_{1} \Rightarrow t_{1}=\frac{m r_{1}^{2} \beta}{k_{1}}
\end{aligned}
$$

which upon combination we get

$$
\left(t_{1}+t_{1}\right)=\tau_{1}=\frac{m(\mu+\beta) r_{1}^{2}}{k_{1}} \Rightarrow \frac{1}{\tau_{1}}=\frac{k_{1}}{m r_{1}^{2}(\mu+\beta)}
$$


Similarly from (2.42) and (2.43) it can be shown that

$\left(t_{2}+t_{2}\right)=\tau_{2}=\frac{m(\phi+\alpha) r_{2}^{2}}{k_{2}} \Rightarrow \frac{1}{\tau_{2}}=\frac{k_{2}}{m r_{2}^{2}(\phi+\alpha)}$

Hence upon the substitution of (2.66) - (2.72) into (2.65), we realize after some simplification that

$$
\begin{gathered}
\langle U\rangle=-\frac{m k}{\tau \ell} \int_{0}^{2 \pi} r d \theta-m g \frac{k_{1}}{m(\mu+\beta) r_{1}^{2}} \int_{0}^{\pi / 2}\left(r_{1} \sin \mu \tan \beta\right) \frac{d r_{1}}{r_{1} \sqrt{2 \ddot{\beta} \tan \beta}} \\
-m g \frac{k_{2}}{m(\phi+\alpha) r_{2}^{2}} \int_{0}^{\pi / 2}\left(r_{2} \sin \phi \tan \alpha\right) \frac{d r_{2}}{r_{2} \sqrt{2 \ddot{\alpha} \tan \alpha}}
\end{gathered}
$$

Now, substituting $\lambda / r=1+\varepsilon \cos \theta$ and $\tau=(2 m \ell) \pi \sqrt{\lambda} a^{3 / 2}$, then the first integral term becomes

$$
\langle U\rangle=-\frac{k \sqrt{\lambda}}{2 \pi a^{3 / 2}} \int_{0}^{2 \pi} \frac{1}{1+\varepsilon \cos \theta} d \theta-\frac{g k_{1}}{(\mu+\beta)} \frac{\sin \mu \tan \beta}{\sqrt{2 \ddot{\beta} \tan \beta}} \int_{0}^{\pi / 2} \frac{1}{r_{1}^{2}} d r_{1}-\frac{g k_{2}}{(\phi+\alpha)} \frac{\sin \phi \tan \alpha}{\sqrt{2 \ddot{\alpha} \tan \alpha}} \int_{0}^{\pi / 2} \frac{1}{r_{2}^{2}} d r_{2}
$$

where $a$ is the semi-major axis of the ellipse $\lambda$ is an arbitrary constant. After a careful evaluation it can be shown by using any known integral technique that the first integral

$$
\int_{0}^{2 \pi} \frac{1}{1+\varepsilon \cos \theta} d \theta=\frac{2 \pi}{\sqrt{1-\varepsilon^{2}}}
$$

with the relation $\lambda=a\left(1-\varepsilon^{2}\right)$ and the substitution of (2.75) into the first term of (2.74), while maintaining that the other two integrals have trivial solutions, then $(2.74)$ reduces to

$$
\langle U\rangle=-\frac{k}{a}+\frac{2 g}{\pi}\left(\frac{k_{1} \sin \mu \tan \beta}{(\mu+\beta) \sqrt{2 \ddot{\beta} \tan \beta}}+\frac{k_{2} \sin \phi \tan \alpha}{(\phi+\alpha) \sqrt{2 \ddot{\alpha} \tan \alpha}}\right)
$$

Thus the potential energy is partly attractive and partly repulsive. Suppose it is only attractive then

$$
\begin{aligned}
& \frac{k_{1} \sin \mu \tan \beta}{(\mu+\beta) \sqrt{2 \ddot{\beta} \tan \beta}}=-\frac{k_{2} \sin \phi \tan \alpha}{(\phi+\alpha) \sqrt{2 \ddot{\alpha} \tan \alpha}} \\
& \frac{k_{1}}{k_{2}}=-\left(\frac{(\mu+\beta) \sin \phi \tan \alpha \sqrt{2 \ddot{\beta} \tan \beta}}{(\phi+\alpha) \sin \mu \tan \beta \sqrt{2 \ddot{\alpha} \tan \alpha}}\right)
\end{aligned}
$$

Hence the newly included potential energy constants of the central force motion as a result of the oscillating phases are oppositely directed.

\subsection{Discussion of Results}

It is clear from the force equation given by (2.8), that the force field is quadratic with respect to the variable angular displacements. The force field is six-dimensional (6D) in character and it comprises of the translational and oscillating phases. The oscillating phase of the force field increases as the orbital spin oscillating angles is increased. Also the translational phase decreases as $\theta, \mu$ and $\phi$ is increased. The net result of this equation would yield a negative force field which is attractive in character.

The upper and lower orbital spin oscillating velocities $\dot{\beta}$ and $\dot{\alpha}$ has two possible roots with positive and negative gradients. This is shown in (2.22) and (2.23). The positive gradient describes the oscillation in the downward direction $D \rightarrow C$, while the negative gradient of $\dot{\beta}$ determines the oscillation in the upward direction $C \rightarrow D$. 
The interpretation is the reverse in the case of $\dot{\alpha}$. The positive gradient root of $\dot{\alpha}$ describes the oscillation in the lower upper direction $B \rightarrow C$, while the negative gradient root of $\dot{\alpha}$ determines the oscillation in the lower downward direction $C \rightarrow B$. The orbital spin oscillating velocities increase as the orbital spin oscillating angles $\beta$ and $\alpha$ is increased. Also the values of the orbital spin oscillating angles determine the fictitious radii velocities $\dot{r}_{1}$ and $\dot{r}_{2}$. According to equation (2.24) and (2.25), the fictitious radii velocity increases as the orbital oscillating angles are increased.

Within the valid intervals of $\beta$ and $\alpha$, the energy conveyed in the spin oscillating phase increases as the orbital oscillating angles is increased. This is shown in equation (2.54). The equation shows that the total energy is quadratic with respect to the three independent radii paths. From the radial energy equation, the energy possessed by the body is directly proportional to the square of the translational elliptic radii velocities and the potential force field is negative. The negative characteristic of the angular momenta and the potential force field in the radial energy equation, further affirms that the motion of the body is attractively translational and oscillatory, and it is always directed towards a central point.

It is obvious from equation (2.63) that the areal velocity is partly constant and partly varies with the vertical oscillating phases. Consequently, the initial value of the areal velocity of a body undergoing a central force motion is increased due to the inclusion of the vertical spin oscillating phases.

According to equation (2.76) the resulting potential energy of the body is attractive in the elliptic plane and repulsive in the vertical oscillating phase of the motion. This is a consequence of the fact that the influence of the gravitational force is tangential to the direction of the central force motion.

\subsection{Conclusion}

In order to make the theory of Central - force motion adequately meaningful, we have in this study extended the theory which has only been that of translational and rotational in the elliptical plane with coordinates $(r, \theta)$, by including fictitious radii and spin oscillations $\left(r_{1}, r_{2} ; \beta, \alpha, \phi, \mu\right)$ about the axis of rotation. The energy conveyed in the spin oscillating phase increases as the orbital oscillating angles above or below the horizon of the elliptical plane is increased. In the absence of the spin oscillating parameters, that is, in the region of the interface $C$, we recover the usual equations of a central-force motion in terms of the total energy, areal velocity and the potential energy, whose motion is only that of translational in the elliptical plane. The energy conveyed in the orbital spin oscillating phase of the motion increases as the orbital spin oscillating angles is increased.

\section{Appendix}

Let us consider the rotational motion of a body of mass $m$ about a fixed origin say, $O$, in an elliptical polar coordinate system. Suppose the same body is also oscillating up and down about its equilibrium position as it translates rotationally round the fixed origin. The body thus possesses translational and rotational elliptical motion with polar coordinates $(r, \theta)$ and tangential spin oscillating motion described by the vertical displacement $C \rightarrow D \rightarrow C \rightarrow B \rightarrow C$ and repeatedly in the $y$-direction. The geometry of the analytical requirements is shown in fig. A.1.

The reader should take note that the oscillation of the body is not out of the elliptical orbit of rotation. Rather the displacement $D$ and $B$ above and below $C$ is very small. The oscillation is still within the limits of the axis of rotation $C$. We have only decided to stretch $D$ and $B$ above and below $C$ considerably enough in order to reveal the geometrical concept required for the analytical calculation.

There are six possible degrees of freedom or generalized coordinates exhibited by the motion body under this circumstance: (i) translational and rotational in the elliptical plane $(r, \theta)$,(ii) the plane of upward oscillations $(\beta, \mu)$ and (iii) the plane of downward oscillations $(\alpha, \phi)$.

We shall compute separately the tangential spin oscillating motions in both oscillating frames and eventually combine the result with the orbital elliptical plane motion. In this study, we assume that the angular displacements in the tangential spin oscillating frames are not equal and so the system under study is not radially symmetric. Consequently, there is the existence of torque due to the non uniformity of the radii distances.

Accordingly, we can now develop relationships between the various areas indicated on fig. A. 1, with the goal to find the formula for the area swept out by the elliptical plane polar motion, and the result obtained from this is then added to the tangential oscillating triangle sections $D \hat{O} C$ and $C \hat{O} B$ respectively.

From the figure, $P$ and $Q$ are very small upward and downward displacements from the equilibrium axis of rotation $C$, that is, regions in the upper and lower triangular swept segments of the upper and lower elliptical plane. Our first task would be to connect all these oscillating spin angular degrees of freedom into an expression in terms of $P$ and $Q$. 
For clarity of purpose, let us define the various symbols which we shall encounter in our calculations : (i) the elliptical radius $r$ (ii) the fictitious radii $r_{1}$ and $r_{2}$ (iii) the plane of upward oscillations $(\beta, \mu)$, that is subtended from the upper elliptical plane (iv) the plane of downward oscillations $(\alpha, \phi)$, that is subtended from the bottom or the lower part of the elliptical plane (v) the elliptical orbital angle $\theta$ (vi) the upper tangential oscillating spin angle $\mu$ (vii) the lower tangential oscillating spin angle $\phi$ (viii) the upper and lower orbital spin oscillating angles $\beta$ and $\alpha$

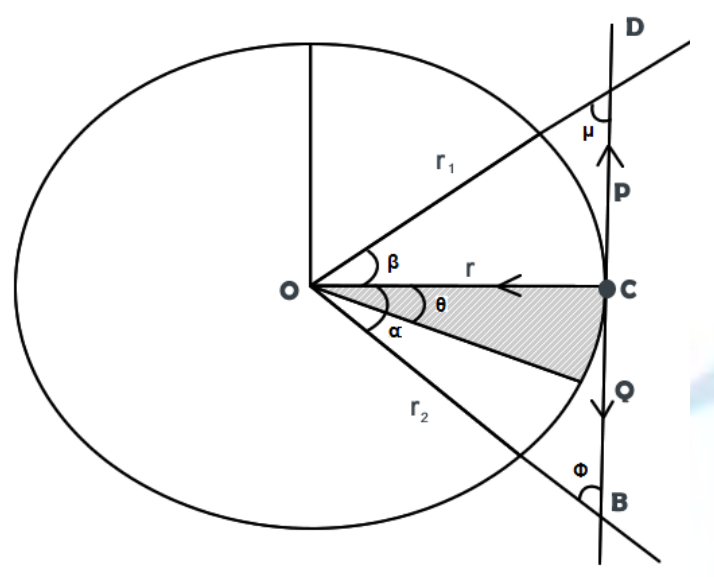

Fig. A.1. Represents the elliptical and oscillating motion of a body in a central-force field. The body is oscillating up and down about the axis of rotation $C$. Where $\triangle D \hat{O} C$ (frame I) and $\triangle C \hat{O} B$ (frame II) are the respective upper and lower projections onto the plane of the ellipse, the lines $\overline{D C}(P)$ and $\overline{C B}(Q)$ are very small displacements from the axis $C$, we have only stretched them to make the geometry of the figure clear enough for observation and calculation.

In frame l: we obtain from $\triangle D \hat{O} C$

$r=r_{1} \sin \mu \quad ; \quad P=r \tan \beta=r_{1} \sin \mu \tan \beta$

In frame II: we obtain from $\triangle C \hat{O} B$

$r=r_{2} \sin \phi \quad ; \quad Q=r \tan \alpha=r_{2} \sin \phi \tan \alpha$

The position vector $\vec{r}$ of the body in the translating and rotating elliptical plane polar orbit is given by

$\vec{r}=x i=r \cos \theta i$

While in combination with the spin oscillating frames (acting in the $y$-direction), we get

$\vec{r}=x i+y j=x i+P j^{\uparrow}+Q j^{\downarrow} \quad ; \quad \vec{r}=r \cos \theta i+r_{1} \sin \mu \tan \beta j^{\uparrow}+r_{2} \sin \phi \tan \alpha j^{\downarrow}$

$\hat{r}=\frac{\partial \vec{r}}{\partial r}=\cos \theta i ; \quad \hat{r}_{1}=\frac{\partial \vec{r}}{\partial r_{1}}=\sin \mu \tan \beta j^{\uparrow} ; \hat{r}_{2}=\frac{\partial \vec{r}}{\partial r_{2}}=\sin \phi \tan \alpha j^{\downarrow}$

$\vec{r}=r \hat{r}(\theta)+r_{1} \hat{r}_{1}(\mu, \beta)+r_{2} \hat{r}_{2}(\phi, \alpha)$

$\hat{\theta}=\frac{\partial \hat{r}}{\partial \theta}=-\sin \theta i \quad ; \quad \frac{\partial \hat{\theta}}{\partial \theta}=-\cos \theta i=-\hat{r}$

$\hat{\mu}=\frac{\partial \hat{r}_{1}}{\partial \mu}=\cos \mu \tan \beta j^{\uparrow} ; \quad \frac{\partial \hat{\mu}}{\partial \mu}=-\sin \mu \tan \beta j^{\uparrow}=-\hat{r}_{1}$ 
$\hat{\beta}=\frac{\partial \hat{r}_{1}}{\partial \beta}=\sin \mu \sec ^{2} \beta j^{\uparrow} \quad ; \quad \frac{\partial \hat{\beta}}{\partial \beta}=2 \tan \beta \sin \mu \sec ^{2} \beta j^{\uparrow}=2 \tan \beta \hat{\beta}$

$\hat{\phi}=\frac{\partial \hat{r}_{2}}{\partial \phi}=\cos \phi \tan \alpha j^{\downarrow} \quad ; \quad \frac{\partial \hat{\phi}}{\partial \phi}=-\sin \phi \tan \alpha j^{\downarrow}=-\hat{r}_{2}$

$\hat{\alpha}=\frac{\partial \hat{r}_{2}}{\partial \alpha}=\sin \phi \sec ^{2} \alpha j^{\downarrow} \quad ; \quad \frac{\partial \hat{\alpha}}{\partial \alpha}=2 \tan \alpha \sin \phi \sec ^{2} \alpha j^{\downarrow}=2 \tan \alpha \hat{\alpha}$

Note that we did not use the same expression for the elliptical radius $r$ in frames I and II, even if it is common to both frames. This is because we want the fictitious radii to feature simultaneously in the general equation for the position vector of the rotating and oscillating body. Besides, it may also be possible that both radii are unequal.

\section{References}

[1]. Alan J. Brizard (2008). "Motion in a Central-Force field"

http://scienceworld.wolfram.com/physics/CentalForce.html.

[2]. Whittaker E. T. (1937) “ Analytical Dynamics” Cambridge, England : Cambridge University Press.

[3]. Meriam J. L. and Kraige.L. G. (2009). Engineering mechanics, DYNAMICS, $5^{\text {th }}$ Edition.

[4]. Rosu L. and Haret C. (1979). "Classical Mechanics” Physics Education. [arxiv.org:physics/9909035]

[5]. Enaibe A. Edison, Agbalagba O. Ezekiel, Johnny A Francis and Nelson Maxwell (2013). "Langranges's Equation of Motion for Oscillating Central-force field". Journal of Theoretical Mathematics and Applications (JTMA), UK. Vol. 3, No. 2, pp99 - 115.

[6]. Enaibe A. Edison, Enukpere E. Emmanuel and John O. A . Idiodi (2013). "The Modified Theory of Central- force Motion". International organization for scientific research (IOSR), Journal of Applied Physics (IOSR - JAP), India. Vol. 4, No. 1, pp $75-82$.

[7]. Keith R. Symon.(1997) "Mechanics", 3rd edition. Addison-Wesley publishing company.

[8]. Weisstein Eric W. (2007). "Central Force" 